\title{
Estudo Comparativo do Uso de Prednisolona versus Acetato de Hidrocortisona no Tratamento da Hiperplasia Adrenal Congênita por Deficiência da 21-Hidroxilase Forma Clássica
}

\begin{abstract}
RESUMO
O tratamento da hiperplasia adrenal congênita (HAC) por deficiência da 21-hidroxilase forma clássica é habitualmente realizado com acetato de hidrocortisona. A hidrocortisona oral, em nosso meio, só está disponível em farmácias de manipulação. A prednisolona possuisolução oral estável, comercialmente disponível, e tem como vantagem poder ser utilizada em dose única diária. 0 objetivo desse estudo foi comparar a eficácia da prednisolona aos resultados obtidos com o acetato de hidrocortisona. Foram estudados 15 pacientes, idade cronológica média (DP) de 7,2 anos $(3,6)$, em dois períodos consecutivos de um ano, inicialmente utilizando a hidrocortisona $\left(17,5 \mathrm{mg} / \mathrm{m}^{2} / \mathrm{dia}\right.$, divididos em três doses), seguida do uso de prednisolona $\left(3 \mathrm{mg} / \mathrm{m}^{2} / \mathrm{dia}\right.$, dose única matinal). A avaliação dos tratamentos foi realizada por meio das variações do escore $Z$ de estatura para idade cronológica ( $\triangle Z E$ ), do escore $Z$ de estatura para a idade óssea ( $\triangle Z E I O)$ e do escore $Z$ do Índice de massa corporal (IMC) ( $\triangle \mathrm{ZIMC)}$, bem como os valores da androstenediona em cada período. Não houve diferença na $\Delta Z E$, na $\Delta Z E I O$ e na $\Delta Z I M C$ entre os dois períodos, assim como nos valores de androstenediona. Concluiu-se que a prednisolona em dose única diária apresenta eficácia semelhante à obtida com a hidrocortisona utilizada três vezes ao dia, podendo ser considerada uma opção terapêutica nos pacientes com HAC por deficiência da 21-hidroxilase. (Arq Bras Endocrinol Metab 2008;52/ 1:101-108)
\end{abstract}

Descritores: Hiperplasia supra-renal congênita; 21-hidroxilase; Prednisolona; Hidrocortisona

\section{ABSTRACT}

Comparative Study of Prednisolone versus Hydrocortisone Acetate for Treatment of Patients with the Classic Congenital Adrenal Hyperplasia due to 21-Hydroxylase Deficiency.

Hydrocortisone acetate is usually employed in the treatment of classic congenital adrenal hyperplasia (CAH) due to 21-hydroxylase deficiency. In Brazil, however, oral hydrocortisone acetate is only available from manipulation pharmacies. Prednisolone has stable oral pharmaceutical formulations commercially available, with the advantage of a single daily dose. The aim of this study was to compare the efficacy of oral prednisolone and oral hydrocortisone in the treatment of $\mathrm{CAH}$ due to 21-hydroxylase deficiency. Fifteen patients with mean (SD) chronological age of $7.2(3.6)$ years, were evaluated in two consecutive 1-year periods. In the first year, hydrocortisone $\left(17.5 \mathrm{mg} / \mathrm{m}^{2} /\right.$ day, divided in three doses) was used in the treatment, followed by the use of prednisolone ( $3 \mathrm{mg} / \mathrm{m}^{2} / \mathrm{day}$, once in the morning) in the second year. The comparison between the two treatments was assessed after a one-year treatment period by: variation of height standard deviation score (SDS) ( $\Delta$ Height SDS), variation of height SDS according to bone age ( $\triangle$ BA SDS), variation of body mass SDS ( $\triangle$ BMI SDS) and serum levels of androstenedione. No significant difference was observed in relation to the $\Delta$ Height SDS, $\Delta$ BA SDS artigo original

\author{
FLAVIA M. LEITE \\ Carlos A. Longui \\ CRISTIANE KOCHI \\ ClaUdIA D. C. FARIA \\ MAURO BORGHI \\ LUIS EDUARDo P. CalLIARI \\ Osmar Monte
}

Unidade de Endocrinologia Pediátrica do Departamento de Pediatria e Puericultura da Irmandade da Santa Casa de Misericórdia de São Paulo, São Paulo, SP, Brasil.

Recebido em 09/03/2007 Aceito em 03/08/2007 
and $\triangle \mathrm{BMI}$ SDS. No significant difference was observed in the serum levels of androstenedione. We conclude that the efficacy of prednisolone administered once a day orally is comparable to the oral use of hydrocortisone three times a day. Oral prednisolone may be an option for patients with $\mathrm{CAH}$ due to 21-hydroxylase deficiency. (Arq Bras Endocrinol Metab 2008;52/1:101-108)

Keywords: Congenital adrenal hyperplasia; 21-hydroxylase; Prednisolone; Hydrocortisone.

\section{INTRODUÇÃO}

\begin{abstract}
DEFICIÊNCIA DA ENZIMA 21-hidroxilase (21OHase) constitui um erro inato do metabolismo dos esteróides adrenais, é geneticamente transmitida com caráter autossômico recessivo e causada por mutação de seu gene codificador - CYP21 (1). A deficiência da enzima 21 OHase é responsável por $90 \%$ dos casos de hiperplasia adrenal congênita (HAC), incidindo entre 1:5.000 e 1:15.000 nascidos vivos e com freqüência de portadores da mutação em 1:60 indivíduos $(2,3)$.

Na HAC por deficiência da enzima 210 Hase, o defeito enzimático determina hipocortisolismo, que por falta de feedback negativo determina aumento da secreção do hormônio adrenocorticotrófico (ACTH), com conseqüente hiperplasia das células do córtex adrenal. Simultaneamente, ocorre produção excessiva de esteróides intermediários prévios ao bloqueio, entre eles os andrógenos adrenais, como a dehidroepiandrosterona (DHEA) e a androstenediona (A), perifericamente convertida à testosterona $(\mathrm{T})$.

A HAC por deficiência da enzima 210 Hase é classificada em duas formas: clássica e não-clássica. A forma clássica, caracterizada por virilização intra-útero no sexo feminino e virilização precoce em ambos os sexos, determina aceleração da velocidade de crescimento e da maturação óssea, com conseqüente perda de estatura final $(4,5)$.

$\mathrm{O}$ tratamento da deficiência da 210 Hase é feito com base na reposição de glicocorticóide (GC), com o objetivo de corrigir a deficiência de cortisol e melhorar a qualidade de vida, bem como reduzir a morbimortalidade associada a essa doença (6,7). Entretanto, esse tratamento classicamente utilizado leva freqüentemente a resultados insatisfatórios (8). Estudos de longa duração demonstram que pacientes com HAC não atingem
\end{abstract}

a estatura-alvo $(\mathrm{TH})$ representativa da estatura familial (9-11). Metanálise de 18 estudos, publicada em 2001 por Eugster e cols., demonstrou que crianças com HAC por deficiência da 210 Hase têm redução média da estatura final de -1,37 DP (12). Problemas adicionais como irregularidade menstrual, infertilidade e distúrbios psicossociais também são comuns (13-16). O uso inadvertido de doses excessivas de GC também tem sido responsabilizado pelo comprometimento da estatura final $(17,18)$.

$\mathrm{O}$ acetato de hidrocortisona, por ser o hormônio mais similar ao cortisol endógeno, é considerado a melhor opção terapêutica, especialmente em crianças, nas quais existe maior risco de supressão do crescimento com o uso de outros GCs sintéticos de ação mais prolongada (19). No entanto, a hidrocortisona possui meia-vida biológica curta, necessitando de intervalo máximo entre as doses de 8 horas. Isto dificulta a adesão ao tratamento e facilita a não-supressão adequada do ACTH, determinando freqüente excesso de produção androgênica. A dose inicial preconizada de hidrocortisona varia entre 10 e $15 \mathrm{mg} / \mathrm{m}^{2} \mathrm{SC}$, dividida em três tomadas ao dia, dose esta pouco acima da secreção fisiológica de cortisol de 8 a $10 \mathrm{mg} / 24$ horas $(20,21)$. Sua apresentação oral não está disponível comercialmente no Brasil, e é obtida por meio da manipulação artesanal do fármaco. A forma líquida é instável (suspensão), apresenta precipitação em diluente não-alcoólico, e é recomendável a utilização de cápsulas ou comprimidos $(22,23)$. Essas características dificultam a administração ou facilitam os erros de manipulação, os erros de dosagem e as falhas determinadas pela necessidade de dissolver o conteúdo das cápsulas para permitir sua ingestão por crianças menores. Essas falhas acarretam freqüentes sub ou superdosagens do fármaco, determinando controle inadequado da doença e efeitos colaterais mais freqüentes e graves (8). 
A adesão ao tratamento é fator indispensável para obtenção de resultados adequados. Por ser uma doença crônica, a HAC necessita tratamento contínuo, e é de fundamental importância a busca de um esquema terapêutico que se adapte ao cotidiano do paciente e de seus responsáveis. A possibilidade de uso de medicação em dose única diária, com emprego de GC com meiavida intermediária tem sido proposta por diversos autores $(8,24-29)$.

A prednisolona, um GC de meia-vida intermediária, tem sido apontada como uma opção terapêutica em crianças e adolescentes com deficiência da 210 Hase $(8,28)$. Trata-se de um GC sintético, estruturalmente semelhante ao cortisol, que tem como única diferença molecular a existência de dupla ligação entre os carbonos 1 e 2. Essa característica prolonga sua meia-vida plasmática e possibilita sua utilização em dose única diária. A dose inicial preconizada é de $3 \mathrm{mg} / \mathrm{m}^{2}$, representando um quarto a um quinto da dose de hidrocortisona. Sua apresentação oral é comercialmente disponível em forma de solução, o que facilita a aquisição e administração às crianças. $O$ custo é menor quando comparado à hidrocortisona formulada. Estudos empregando esse fármaco no tratamento da HAC têm demonstrado eficiência equivalente à hidrocortisona $(8,26)$.

Neste estudo, avaliou-se a eficácia do tratamento com prednisolona em dose única diária pela manhã no controle clínico-laboratorial de pacientes com HAC por deficiência da 21-OHase comparado aos resultados observados nos mesmos pacientes que utilizaram previamente o acetato de hidrocortisona.

\section{CASUÍSTICA E MÉTODOS}

Foram avaliados 15 pacientes com diagnóstico de HAC por deficiência da 210 Hase, acompanhados na Unidade de Endocrinologia Pediátrica da Irmandade da Santa Casa de Misericórdia de São Paulo. Destes, 14 apresentavam a forma clássica perdedora de sal, sendo nove do sexo feminino e cinco do sexo masculino. Uma paciente apresentava a forma clássica virilizante simples. A idade cronológica variou de 1,7 a 13,1 anos, com mediana de 6,8 anos. Dez pacientes eram pré-púberes (Ml e G1) e cinco púberes (M2-4 e G2-4), segundo a classificação de Tanner e cols. (30).

O protocolo de estudo foi aprovado pelo Comitê de Ética em Pesquisa da Instituição e iniciado após a obtenção do termo de consentimento, assinado por um dos pais ou responsável pela criança.

A avaliação foi feita a cada três meses, e dividida em dois períodos distintos com um ano de duração cada. No primeiro ano (tempo - 12:0), utilizou-se o acetato de hidrocortisona, sendo a análise dos dados realizada de forma retrospectiva. No segundo ano, empregou-se a prednisolona (tempo $-0:+12$ ) e a coleta dos dados foi realizada de forma prospectiva (Figura 1 ).

A dose média da hidrocortisona utilizada no início da avaliação foi de $17,5 \mathrm{mg} / \mathrm{m}^{2} /$ dia dividida em três doses diárias $(8 \mathrm{~h}, 14 \mathrm{~h}$ e $20 \mathrm{~h})$, sendo a maior dose pela manhã $(50 \%, 25 \%, 25 \%$ da dose total diária); na época da troca para prednisolona, denominado tempo 0 (zero), a dose de hidrocortisona era de $16,6 \mathrm{mg} / \mathrm{m}^{2} /$ dia. A mesma foi substituída pela prednisolona $3 \mathrm{mg} /$ $\mathrm{m}^{2} /$ dia em dose única diária pela manhã. Mineralocorticóide (9- $\alpha$-fluodrocortisona) foi utilizado nos pacien-

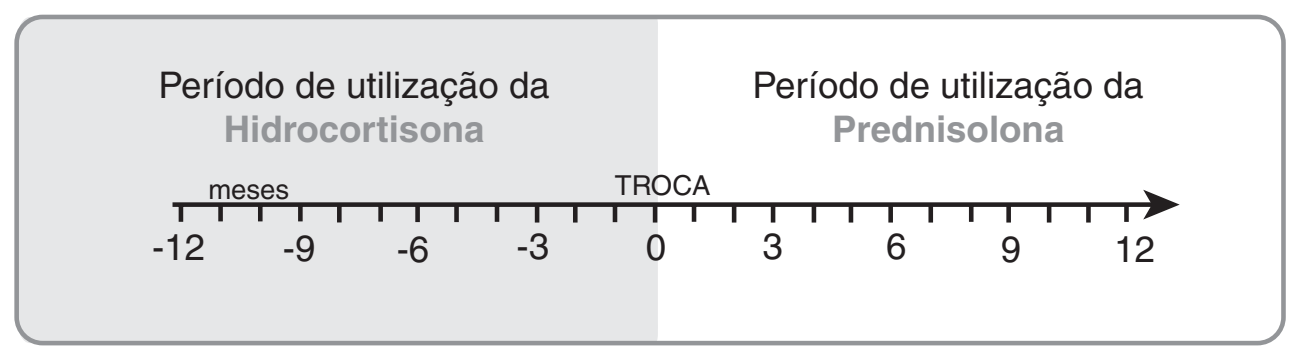

Figura 1. Protocolo utilizado na avaliação dos pacientes durante o uso da hidrocortisona e após sua troca pela prednisolona

Avaliação trimestral dos pacientes. Meses analisados retrospectivamente: números negativos (durante tratamento com hidrocortisona); meses analisados prospectivamente: números positivos (durante tratamento com prednisolona). Tempo 0 (zero): momento da troca das medicações. 
tes com FPS na dose de 50 a $100 \mu \mathrm{g} /$ dia não sendo modificada durante o estudo.

Os pacientes foram avaliados em consulta ambulatorial a cada três meses, sendo descritas as variáveis clínicas: peso, estatura, estádio puberal, sinais de virilização e/ou hipercortisolismo. A avaliação laboratorial trimestral incluiu a quantificação da $17 \mathrm{OHP}$ e da $\mathrm{A}$. $\mathrm{O}$ método empregado na quantificação hormonal foi o radioimunoensaio, utilizando-se kits da DSL (Diagnostic Systems Laboratories, Webster, Texas, Estados Unidos).

Radiografias de mão e punho foram solicitadas a cada seis meses para avaliação da idade óssea, a qual foi estimada pelo método de Greulich-Pyle (31).

Quando necessário, a dose da prednisolona era ajustada em $1 \mathrm{mg} / \mathrm{m}^{2} \mathrm{SC}$, procurando manter valores adequados de androstenediona. A dose média da prednisolona utilizada ao final do estudo foi de $4,73 \mathrm{mg} /$ $\mathrm{m}^{2}$, variando entre 3 e $6 \mathrm{mg} / \mathrm{m}^{2}$.

A estatura foi ajustada para a idade e o sexo e expressa em número de desvios-padrão (escore $\mathrm{Z}=\mathrm{ZE}$ ). O mesmo foi feito para o índice de massa corpórea (ZIMC) e para a estatura corrigida para a idade óssea (ZEIO). Essas variáveis foram avaliadas nos tempos: um ano antes (-12 meses) quando em uso de hidrocortisona, no momento da troca pela prednisolona (tempo 0 ) e após um ano de uso da prednisolona (+12 meses). Para o cálculo das variáveis utilizou-se o programa Growth Analyser (Dutch Growth Foundation, 2000-2006), tendo como referência os padrões do NCHS-2000 (U.S. Departament of Health and Human Service. Centers for Disease Control and Prevention. National Center for Health Statistics) $(32,33)$.

Para cada uma das variáveis, foi adicionalmente calculada sua variação $(\Delta)$ em cada período de tratamento: $\Delta \mathrm{ZE}, \Delta \mathrm{ZIMC}, \Delta \mathrm{ZEIO}$.

Para a avaliação de $17 \mathrm{OHP}$ e A, foi calculada a média dos valores observados em cada período (média de duas a quatro determinações). Na avaliação da $17 \mathrm{OHP}$ também foi utilizada a mediana dos valores nos dois períodos, uma vez que grande parte dos resultados foram superiores ao limite máximo de detecção $(20 \mathrm{ng} / \mathrm{mL})$.

Diante da grande variabilidade dos valores hormonais observados no controle laboratorial dos pacientes, adicionamos uma segunda ferramenta para avaliar o grau de controle da doença, classificando-se de forma aleatória como bom, regular ou mau controle de acordo com os valores da A e da 170HP nos diferentes períodos de tratamento (15).
Considerou-se bom controle quando a $17 \mathrm{OHP}$ permanecia $<10 \mathrm{ng} / \mathrm{mL}$ em mais de $75 \%$ dos resultados determinados no período. Regular quando a $17 \mathrm{OHP}$ permaneceu $<10 \mathrm{ng} / \mathrm{mL}$ entre $25 \%$ e $75 \%$ dos resultados no período avaliado e mau controle quando a $17 \mathrm{OHP}$ permaneceu $<10 \mathrm{ng} / \mathrm{mL}$ em menos de $25 \%$ dos resultados do período analisado. Em relação à $\mathrm{A}$, considerou-se bom controle quando permaneceu $<2 \mathrm{ng} / \mathrm{mL}$ em mais de $75 \%$ dos resultados obtidos no período; regular quando permaneceu $<2 \mathrm{ng} / \mathrm{mL}$ entre $25 \%$ e $75 \%$ dos resultados e mau controle quando permaneceu $<2 \mathrm{ng} / \mathrm{mL}$ em menos de $25 \%$ dos resultados obtidos no período analisado.

A análise estatística das variáveis foi realizada utilizando-se o programa estatístico SigmaStat. for Windows Version 2.03 (SPSS Inc. Headquarters, 1997). O teste pareado não-paramétrico (Wilcoxon) foi utilizado para comparar os valores da 17OHP e da A entre os dois períodos. $\mathrm{O}$ teste de análise de variância para amostras repetidas (ANOVA for repeated measures) foi utilizado para avaliar as variações $\Delta \mathrm{ZE}, \Delta \mathrm{ZIMC}$ e $\Delta \mathrm{ZEIO}$ entre os três diferentes momentos $(-12$ meses; tempo $0 ;+12$ meses). Considerou-se diferença significante quando os valores de $\mathrm{p}<0,05$.

\section{RESULTADOS}

Os pacientes foram avaliados clínica e laboratorialmente um ano antes da troca da medicação enquanto faziam uso de hidrocortisona (-12 meses), no momento da substituição (tempo 0) e após um ano com a prednisolona (+12 meses). A variação dos valores observados entre os períodos é descrita na tabela 1 .

Foi identificado aumento significativo no ZE após um ano de uso da prednisolona (+12 meses) em relação ao ZE no início do estudo (-12 meses), porém não houve diferença entre ZE no início do estudo (-12 meses) em relação ao ZE no momento da troca (tempo 0) ou quando este foi comparado ao ZE após a prednisolona (+12 meses). Não houve diferença significante entre os períodos na avaliação do ZIMC.

Houve diminuição progressiva do $\mathrm{Z}$ de estatura para idade óssea entre os períodos - 12 meses e +12 meses, porém não houve diferença quando o ZEIO do início do estudo (-12) foi comparado com o ZEIO no momento da troca $(0)$ e este com o ZEIO após o uso da prednisolona $(+12)$. 
Tabela 1. Valor médio (DP) e mediana de estatura, índice de massa corporal (IMC) e valores hormonais em pacientes com hiperplasia adrenal congênita forma clássica tratados com acetato de hidrocortisona e subseqüentemente com prednisolona

\begin{tabular}{lccc}
\hline & $\begin{array}{c}\text { Hidrocortisona } \\
(-12) \text { meses }\end{array}$ & $\begin{array}{c}\text { Tempo } 0 \\
\text { troca fármaco }\end{array}$ & $\begin{array}{c}\text { Prednisolona } \\
(+12) \text { meses }\end{array}$ \\
\hline Estatura $(\mathrm{cm})$ & $113,5(23,9)$ & $122,0(21,7)$ & $129,9(21,3)$ \\
\hline ZE & $-0,1(1,3)^{a}$ & $0,1(1,3)$ & $0,3(1,3)^{a}$ \\
ZEIO & $-0,7^{b}$ & $-1,0$ & $-1,3^{b}$ \\
\hline IMC $\left(\mathrm{kg} / \mathrm{m}^{2}\right)$ & $17,4(2,2)$ & $17,2(1,7)$ & $17,5(2,2)$ \\
\hline ZIMC & $0,5(1,1)$ & $0,5(1,1)$ & $0,4(0,1)$ \\
\hline A $(\mathrm{ng} / \mathrm{mL})$ & $1,9(1,5)$ & n.a. & $1,9(1,3)$ \\
\hline $170 H P(\mathrm{ng} / \mathrm{mL})$ & $14,3(5,5)^{c}$ & n.a. & $18,1(2,9)^{c}$ \\
\hline
\end{tabular}

$\mathrm{A}=$ androstenediona - valores médios observados no período; 170HP: 17-hidroxiprogesterona - valores médios observados no período; $\mathrm{a}=$ hidrocortisona (-12) versus tempo 0 versus prednisolona (+12); $\mathrm{p}=<0,05$; One way repeated measures analysis of variance; $\mathrm{b}=$ hidrocortisona (-12) versus tempo 0 versus prednisolona $(+12) ; \mathrm{p}=<$ 0,05; Friedman repeated measures analysis of variance on ranks; $\mathrm{C}=$ hidrocortisona (-12) versus prednisolona (+12); $\mathrm{p}=<0,05$; Teste pareado não-paramétrico (Wilcoxon).

Considerando o ZEIO como critério de previsão de estatura final dos pacientes, houve perda progressiva de previsão de estatura, sem diferença quanto ao grau de perda quando analisados os dois períodos de tratamento. Quando a previsão de estatura final feita com base no ZEIO foi comparada à estatura-alvo $(\mathrm{ZTH})$, que para estes pacientes era de - $0,52(0,6)$, observou-se uma provável perda de -1 DP ao final do estudo.

Houve aumento da média dos valores da $17 \mathrm{OHP}$ entre os dois períodos, porém não houve diferença quando avaliada a mediana da $17 \mathrm{OHP}$ nesses períodos. Não houve diferença entre a média dos valores da androstenediona (Tabela 1).

Quando empregado o critério de classificação do controle laboratorial como bom, regular ou mau, ao se avaliarem os valores de $17 \mathrm{OHP}$ durante a utilização do HC, 8/15 pacientes permaneceram mal controlados, porém, ao avaliar a $\mathrm{A}, 9 / 15$ permaneceram com bom controle e 4/15 regulares; já com a prednisolona, 13/15 permaneceram mal controlados conforme os valores da $17 \mathrm{OHP}$, porém tiveram igualmente de bom $(9 / 15)$ para regular $(6 / 15)$ controle quando analisados os valores da androstenediona.

\section{DISCUSSÃo}

A HAC por deficiência da 21-hidroxilase é uma doença endócrina com manifestações clínicas resultantes da produção deficiente de cortisol e excessiva de andrógenos (8). O controle terapêutico costuma ser variável, exigindo constante monitoramento (34). A má adesão ao tratamento é comum, gerando protocolos terapêuticos alternativos, como os que incluem a associação de bloqueadores da síntese ou da ação androgênica e estrogênica (3), os quais têm o inconveniente de utilizar múltiplos medicamentos e terem custo elevado. Alternativas terapêuticas mais simples e de maior conveniência também têm sido propostas, permitindo melhor adaptação ao estilo de vida do paciente.

A hidrocortisona, apesar de ser o GC de escolha no tratamento da HAC, possui diversos inconvenientes envolvendo sua apresentação, sua formulação e sua disponibilidade farmacêutica (22). Adicionalmente, tanto o metabolismo quanto o clearance da hidrocortisona são mais rápidos nos pacientes com HAC, quando comparados aos controles (35). Estudos prévios com a prednisolona sugerem sua eficácia no controle do hiperandrogenismo adrenal e na obtenção de crescimento adequado em crianças $(8,25,36)$. Este estudo teve a peculiaridade de avaliar o mesmo paciente, submetido subseqüentemente à utilização da hidrocortisona seguida de prednisolona.

O tratamento com a prednisolona, assim como outros glicocorticóides de meia-vida mais longa que a hidrocortisona, tem sido utilizado com cautela em crianças pelo maior risco de supressão do crescimento (37). Es- 
tudos anteriores, que descreveram supressão do crescimento, utilizaram maior dose de prednisolona em relação à utilizada neste estudo e a administração foi realizada mais de uma vez ao dia $(28,38,39)$.

A estatura dos pacientes no início do estudo, quando faziam uso da hidrocortisona, era adequada para idade cronológica e sexo, porém inadequadamente baixa quando ajustada para a idade óssea. Essa característica manteve-se durante o ano de uso de hidrocortisona, bem como após um ano de tratamento com prednisolona. A desproporção entre o aumento do ZE e o avanço de idade óssea reflete um persistente hiperandrogenismo. Como resultado, o uso de prednisolona não foi capaz de reduzir a perda estatural já presente durante o uso da hidrocortisona, mas não agravou essa perda durante sua utilização, fazendo que se mantivesse a redução da estatura prevista durante os dois períodos de tratamento.

Quando a previsão de estatura final dos pacientes é comparada ao Z da estatura-alvo dos familiares ( $\mathrm{ZTH})$, os estudos previamente descritos apontam desde ausência até moderada perda estatural, entre - 1 e - 2 SD abaixo do $\mathrm{TH}(9,12,40,41)$. Estudo brasileiro realizado com pacientes tratados com hidrocortisona também constatou perda de estatura final (-1,57 SD) com comprometimento em relação à estatura-alvo familiar em torno de -0,72 SD (10). Neste estudo, considerando o ZEIO como critério de previsão de estatura final, observou-se que a variação em torno da estatura-alvo foi de -1,0 DP no final do período de seguimento.

A perda da estatura final associada ao tratamento da HAC relaciona-se ao efeito combinado das reações adversas dos glicocorticóides sobre o crescimento e da supressão inadequada da secreção androgênica (11,40-43).

Os resultados deste estudo demonstram que a prednisolona, administrada uma vez ao dia, determina, em pacientes com mais de 2 anos de idade, um padrão de crescimento semelhante ao obtido com a hidrocortisona administrada três vezes ao dia, provavelmente também relacionado às doses relativamente baixas utilizadas.

Com um ajuste feito a cada três meses (aumento de $1 \mathrm{mg} / \mathrm{m}^{2}$ por vez, ajuste de $30 \%$, semelhante ao habitualmente realizado com a hidrocortisona), ao final do estudo, a dose média empregada de prednisolona foi $4,73 \mathrm{mg} / \mathrm{m}^{2}$, em dose única pela manhã, com uma variação entre 3 e $6 \mathrm{mg} / \mathrm{m}^{2}$. A diferença entre as doses possivelmente reflete a variabilidade individual na absorção e no metabolismo do glicocorticóide bem como a sensibilidade individual (44).
A prednisolona, assim como outros GC, necessita de ajuste cuidadoso de dosagem. Uma vez que os sinais clínicos não são evidentes em curto prazo de avaliação, o controle terapêutico para a deficiência da 210 Hase inclui a avaliação de esteróides séricos, como a 17-hidroxiprogesterona e a androstenediona. Entretanto, a interpretação da $17 \mathrm{OHP}$ deve ser cuidadosa, uma vez que a mesma possui variação circardiana substancial e a tentativa de normalizar sua concentração pode induzir ao uso excessivo de GC (34,45-47). Neste estudo, a quantificação da $17 \mathrm{OHP}$ permaneceu anormalmente elevada em ambos os protocolos de tratamentos, sendo pouco maior no período em que se utilizou a prednisolona. Por outro lado, a androstenediona, utilizada como principal indicador do controle laboratorial (48), permaneceu adequada e de forma semelhante para a prednisolona e hidrocortisona. Esses achados confirmam a baixa eficácia da $170 \mathrm{HP}$ como indicador do grau de controle do hiperandrogenismo no tratamento da HAC sendo útil, porém, para indicar dose excessiva do GC quando os valores de $17 \mathrm{OHP}$ estão suprimidos para o método (11). Considerando valores normais de A como um controle androgênico adequado associado à estabilização da velocidade de crescimento e controle da idade óssea, resultados similares foram obtidos com a administração de prednisolona ou hidrocortisona.

No período em que se utilizou a prednisolona, não se observou nenhum efeito colateral ou aumento de peso, avaliado pela variação do IMC. Os pais ou responsáveis referiram maior facilidade de adesão por ser dose única diária e pela apresentação em solução ser mais simples de administrar às crianças. $\mathrm{O}$ custo da prednisolona também é menor quando comparado à hidrocortisona (8), fato este relevante no que se refere à adesão ao tratamento em relação à realidade socioeconômica de nossa população.

A prednisolona é o GC sintético que estruturalmente mais se assemelha à hidrocortisona. Em nosso estudo, a dose única diária da prednisolona provou ser semelhante à hidrocortisona três vezes ao dia no controle clínico e laboratorial do hiperandrogenismo de pacientes com deficiência da 210 Hase; a posologia única diária de menor custo, mais conveniente e facilitadora de maior adesão ao tratamento. A apresentação da prednisolona em solução é estável e comercialmente disponível, o que facilita a administração, a aquisição e a titulação das doses.

Em virtude da longa experiência e da segurança de uso por mais de 50 anos, a cortisona e a hidrocortisona 
são consideradas o tratamento de escolha da HAC $(19,49)$. Entretanto, nosso estudo mostrou ser a prednisolona tão efetiva quanto a hidrocortisona, podendo ser considerada uma opção segura no tratamento de pacientes com hiperplasia adrenal congênita.

\section{AGRADECIMENTOS}

Agradecemos aos colegas da Unidade de Endocrinologia Pediátrica pela colaboração na captação e no registro de dados.

\section{REFERÊNCIAS}

1. White PC, New MI, Dupont B. Structure of human steroid 21-hidroxylase genes. Proc Natl Acad Sci USA. 1986;83:5111-5.

2. Donohoue PA, Parker K, Migeon CJ. Congenital adrenal hyperplasia. In: Scriver CR, Beaudet AI, Sly WS, Valle D (eds.). The metabolic and molecular bases of inherited disease. 7.ed. New York: McGraw-Hill; 1995. p.2929-66.

3. Merke DP, Bornstein SR, Ávila NA, Chrousos GP. Future directions in the study and management of congenital adrenal hyperplasia due to 21-hydroxylase deficiency. Ann Intern Med. 2002;136:320-34.

4. New MI, Lorenzen F, Lerner AJ, Kohn B, Oberfield SE, Pollack MS, et al. Genotyping steroid 21-hydroxylase deficiency: hormonal reference data. J Clin Endocrinol Metab. 1983;57:320-6.

5. Menezes Filho HC, Dichtchekenian V, Kuperman H, Della Manna T, Damiani D, Setian N. Fatores que interferem no crescimento e na altura final de pacientes com hiperplasia congênita das supra-renais por deficiência da 21-hidroxilase. Arq Bras Endocrinol Metab. 2001;45:520-32.

6. Wilkins L, Lewis RA, Klein R, Gardner LI, Crigler Jr JF, Rosemberg $E$, et al. Treatment of congenital adrenal hyperplasia with cortisone. J Clin Endocrinol Metab. 1951;11:1-25.

7. Bartter FC, Albright F, Forbes AP, Leaf A, Dempsey E, Carroll E. The effects of adrenocorticotropic hormone and cortisone in the adrenogenital syndrome associated with congenital adrenal hyperplasia: An attempt to explain and correct its disordered hormonal pattern. J Clin Invest. 1951;30:237-51.

8. Caldato MCF, Fernandes VT, Kater CE. One year clinical evaluation of single morning dose prednisolone therapy for 21-hydroxylase deficiency. Arq Bras Endocrinol Metab. 2004;48:705-12.

9. Hargitai G, Solyom J, Battelino T, Lebl J, Pribilincova Z, Hauspie $R$, et al. Growth patterns and final height in congenital adrenal hyperplasia due to classical 21-hydroxylase deficiency. Results of a multicenter study. Horm Res. 2001;55:161-71.

10. Lemos-Marini SHV, Guerra-Júnior G, Morcillo AM, Baptista MTM, Silva LO, Maciel-Guerra AT. Congenital adrenal hyperplasia due to 21-hydroxylase deficiency: final height in $27 \mathrm{pa}$ tients with the classical form. Arq Bras Endocrinol Metab. 2005;49:902-7.

11. Bachega TASS. Fatores que podem predizer a estatura final na deficiência da 21-hidroxilase. Arq Bras Endocrinol Metab. 2005;49:867-9.
12. Eugster EA, Dimeglio LA, Wright JC, Freidenberg GR, Seshadri $\mathrm{R}$, Pescovitz $\mathrm{OH}$. Height outcome in congenital adrenal hyperplasia caused by 21-hydroxylase deficiency: a metaanalysis. J Pediatr. 2001;138:26-32.

13. Holmes-Walker DJ, Conway GS, Honour JW, Rumsby G, Jacobs HS. Menstrual disturbance and hypersecretion of progesterone in women with congenital adrenal hyperplasia due to 21-hydroxylase deficiency. Clin Endocrinol. 1995;43:291-6.

14. Berenbaum SA. Effects of early androgens on sex-typed activities and interests in adolescents with congenital adrenal hyperplasia. Horm Behav. 1999;35:102-10.

15. Bachega TASS, Madureira G, Brenlha EML, Ueti RC, Inácio $M$, Denis FT, et al. Tratamento da hiperplasia supra renal congênita por deficiência da 21 hidroxilase. Arq Bras Endocrinol Metab. 2001;45:64-72.

16. Jaaskefainen J, Tiffinen A, Voutilainen R. Sexual function and fertility in adult females and males with congenital adrenal hyperplasia. Horm Res. 2001;56:73-80.

17. Migeon CJ, Donohoue PA. Congenital adrenal hyperplasia caused by 21-hydroxylase deficiency. Its molecular basis and its remaining therapeutic problems. Endocrinol Metab Clin North Am. 1991;20:277-96.

18. Miller WL. Clinical review 54: Genetics, diagnosis, and management of 21-hydroxylase deficiency. J Clin Endocrinol Metab. 1994;78:241-5.

19. Joint LWPES/ESPE CAH Working Group. Consensus statement on 21-hydroxylase deficiency from the Lawson Wilkins Pediatric Endocrine Society and the European Society for Paediatric Endocrinology. J Clin Endocrinol Metab. 2002;87:4048-53.

20. Linder BL, Esteban NV, Yergey AL, Winterer JC, Loriaux DL, Cassorla F. Cortisol production rate in childhood and adolescence. J Pediatr. 1990;117:892-6.

21. Kerrigan JR, Veldhuis JD, Leyo SA, Iranmanesh A, Rogol AD. Estimation of daily cortisol production and clearance rates in normal pubertal males by deconvolution analysis. J Clin Endocrinol Metab. 1993;76:1505-10.

22. Merke DP, Cho D, Calis KA, Keil MF, Chrousos GP. Hydrocortisone suspension and hydrocortisone tablets are not bioequivalent in the treatment of children with congenital adrenal hyperplasia. J Clin Endocrinol Metab. 2001;86:441-5.

23. Charmandari E, Johnston A, Brook CG, Hindmarsh PC. Bioavailability of oral hydrocortisone in patients with congenital adrenal hyperplasia due to 21-hydroxylase deficiency. J Endocrinol. 2001;169:65-70.

24. Hayek A, Crawford JD, Bode HH. Single dose of dexamethasone in treatment of congenital adrenocortical hyperplasia. Metabolism. 1971;20:897-901.

25. Huseman CA, Varma MM, Blizzard RM, Johanson A. Treat ment of congenital virilizing adrenal hyperplasia patients with single and multiple daily doses of prednisone. J Pediatr. 1977;90:538-42.

26. Punthakee Z, Legault L, Polychronakos C. Prednisolone in the treatment of adrenal insufficiency: $A$ re-evaluation of relative potency. J Pediatr. 2003;143:402-5.

27. Richards GE, Grumbach MM, Kaplan SL, Conte FA. The effect of long acting glucocorticoids on menstrual abnormalities in pacients with virilizing congenital adrenal hyperplasia. J Clin Endocrinol Metab. 1978;47:1208-15.

28. Zipf WB, Bacon GE, Kelch RP. Hormonal and clinical responses to prednisone treatment in adolescents with congenital adrenal hyperplasia. Horm Res. 1980;12:206-17. 
29. Rivkees SA, Crawford JD. Dexamethasone treatment of virilizing congenital adrenal hyperplasia: the ability to achieve normal growth. Pediatrics 2000;106:767-73.

30. Tanner JM, Whitehouse RH, Takaishi M. Standards from birth to maturity for height, weight, height velocity and weight velocity. British children,1965. Part II. Arch Dis Child. 1966;41:613-35.

31. Greulich WW, Pyle SI. Radiographic atlas of skeletal development of the hands and wrists. Stanford: Stanford University Press; 1959.

32. Dutch Growth Foundation. [on line] Growth analyzer. Rotterdam, The Netherlands; 2000-2006. Disponível em: http://www. growthanalyser.org/site/Default.php. [2006 May 25].

33. U.S. Department of Health and Human Service. Centers for Disease Control and Prevention. National Center for Health Statistics. [on line] May 2000 CDC growth charts for United States: methods and development. Vital and health statistics. Hyattsville, MD; 2002. Disponível em: http://www.cdc.gov/ nchs/data/series/sr 11/sr11 246.pdf. [2006 May 25].

34. Hughes IA, Dyas J, Robinson J, Walker RF, Fahmy DR. Monitoring treatment in congenital adrenal hyperplasia. Use of serial measurements of $170 \mathrm{H}$-progesterone in plasma, capillary blood, and saliva. Ann N Y Acad Sci. 1985;458:193-202.

35. Groschl M, Rauh M, Dorr H. Cortisol and 17-hydroxyprogesterone kinetics in saliva after oral administration of hydrocortisone in children and young adolescents with congenital adrenal hyperplasia due to 21-hydroxylase deficiency. J Clin Endocrinol Metab. 2002;87:1200-4.

36. Caldato MCF. Vantagem da prednisolona em dose única diária no tratamento da deficiência de 21-hidroxilase: avaliação clínica e cronobiológica. 2003. Tese (Doutorado). São Paulo: Universidade Federal de São Paulo; 2003.

37. Merke DP, Cutler Jr GB. New ideas for medical treatment of congenital adrenal hyperplasia. Endocrinol Metab Clin North Am. 2001;30:121-35.

38. Laron Z, Pertzelan A. The comparative effect of 6 alfa fluroprednisolone, 6 alfa metylprednisolone, and hydrocortisone on linear growth of children with congenital adrenal virilism and Addison's disease. J Pediatr. 1968;73:774-82.

39. Stempfel R, Sheikholislam B, Lebovitz H, Allen E, Franks R. Pituitary growth hormone suppression with low dosage, long acting corticoid administration. J Pediatr. 1968;73:767-73.
40. Brook CG, Zachmann M, Prader A, Murset G. Experience with long term therapy in congenital adrenal hyperplasia. J Pediatr. 1974;85:12-9.

41. David M, Sempe M, Blanc M, Nicolino M, Forest MG, Morel Y. Final height in 69 patients with congenital adrenal hyperplasia due to 21- hydroxylase deficiency. Arch Pediatr. 1994;1:363-7.

42. Stikkelbroeck N, Van't Hof- Grootenboer BA, Hermus AR, Otten BJ, Van't Hof M. Growth inhibition by glucocorticoid treatment in salt wasting 21-hydroxylase deficiency: In early infancy and (pre)puberty. J Clin Endocrinol Metab. 2003;88: 3525-30.

43. Styne DM, Richard GE, Bell JJ. Correlation of glucocorticoid therapy with stature. In: Lee PA, Plotnik LP, Kowarski AA (eds.). Congenital adrenal hyperplasia. Baltimore: University Park Press; 1977. p.247-64.

44. Nichols T, Nugent CA, Tyler FH. Diurnal variation in suppression of adrenal function by glucocorticoids. J Clin Endocrinol Metab. 1965;25:343-9.

45. Bode HH, Rivkees SA, Cowley DM, Pardy K, Johnson S. Home monitoring of 17 hydroxyprogesterone levels in congenital adrenal hyperplasia with filter paper blood samples. J Pediatr. 1999;134:185-9.

46. Winterer J, Chrousos GP, Loriaux DL, Cutler GBJr. Effect of hydrocortisone, dose schedule on adrenal steroid secretion in congenital adrenal hyperplasia. Ann N Y Acad Sci. 1985;458:182-92.

47. Frisch H, Parth K, Schober E, Swoboda W. Circadian patterns of plasma cortisol, 17-hydroxyprogesterone, and testosterone in congenital adrenal hyperplasia. Arch Dis Child. 1981;56:208-13.

48. Bachega TA, Billerbeck AE, Madureira G, Marcondes JA, Longui CA, Leite MV, et al. Molecular genotyping in Brazilian patients with classical and nonclassical forms of 21-hydroxylase deficiency. J Clin Endocrinol Metab. 1998;83:4416-9.

49. White PC, Speiser PW. Congenital adrenal hyperplasia due to 21-hydroxylase deficiency. Endocr Rev.2000;21:245-91.

\section{Endereço para correspondência:}

Carlos Alberto Longui

R. Prof. Dr. Arthur Ramos, 96, $2^{\circ}$ andar

01454-010 São Paulo, SP

E-mail: carloslongui@msn.com 